\title{
SÍNTESE ELETROQUÍMICA E TRATAMENTO DA NUCLEAÇÃO DO FILME DE POLIANILINA (PANI) EM AÇO INOX 304
}

\author{
ELECTROCHEMICAL SYNTHESIS AND \\ NUCLEATION TREATMENT OF POLYANILINE FILM \\ (PANI) 304 IN STAINLESS STEEL
}

\author{
Álvaro Fontana (PG)* \\ Felipe Garcia (IC) \\ Jarem Raul Garcia (PQ)
}

\begin{abstract}
RESUMO
O presente artigo descreve o crescimento de filme de polianilina sobre aço inox $304 \mathrm{em}$ meio de ácido sulfúrico $0,5 \mathrm{~mol} \mathrm{~L}^{-1}$ e anilina $0,2 \mathrm{~mol} \mathrm{~L}^{-1}$ utilizando a técnica de deposição potenciostática a diferentes potenciais usando como contra-eletrodo $\mathrm{Ag} / \mathrm{AgCl}$. O tratamento matemático de NCS (NucleaçãoCrescimento-Superposição) foi à técnica escolhida para o estudo do mecanismo de crescimento dos filmes de polianilina formados sobre a superfície do aço inox 304. O tratamento foi sugerido para um processo de nucleação múltiplo, que podem ser instantâneas ou progressivas, e o crescimento tridimensional de cada núcleo individual controlado por difusão. O mecanismo de nucleação do filme de polianilina na liga de aço inox 304 é do tipo instantânea.
\end{abstract}

Palavras-chave: Polianilina. Nucleação. Cronoamperometria.

\begin{abstract}
This article describe the growth of polyaniline film on 304 stainless steel in sulfuric acid medium $0.5 \mathrm{~mol} \mathrm{~L}^{-1}$ and aniline $0.2 \mathrm{~mol} \mathrm{~L}^{-1}$ utilizing the potentiostatic deposition technique at different outputs and using $\mathrm{Ag} / \mathrm{AgCl}$ as the counter electrode. The NGS (Nucleation-Growth-Superposition) mathematical technique was chosen to study the growth mechanism of the polyaniline film formed on the surface of 304 stainless steel. The treatment was suggested for a multiple nucleation process, which can be instantaneous or progressive, and with the three-dimensional growth of each individual nucleus controlled by diffusion. The nucleation mechanism of polyaniline film on the alloy of 304 stainless steel is instantaneous.
\end{abstract}

Keywords: Polyaniline. Nucleation. Chronoamperometry.

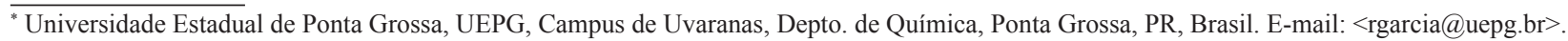




\section{Introdução}

Embora a polianilina (PAni) tenha sido descoberta há aproximadamente 150 anos foi somente na década de 80 que um grande interesse foi depositado a esse polímero e seus derivados como, por exemplo, um material condutor de eletricidade (BAZITO, 2002). Desde então esse material passou a ser estudado mundialmente por inúmeros grupos de pesquisa, devido à sua boa estabilidade química em condições ambientais, relativa facilidade de síntese e dopagem, baixo custo e possibilidade de ampla aplicação tecnológica (MATTOSO, 1996). As polianilinas representam uma classe de polímeros cuja composição química na forma básica é dada pela fórmula representada abaixo.

Figura 1 - Estrutura química geral da Polianilina

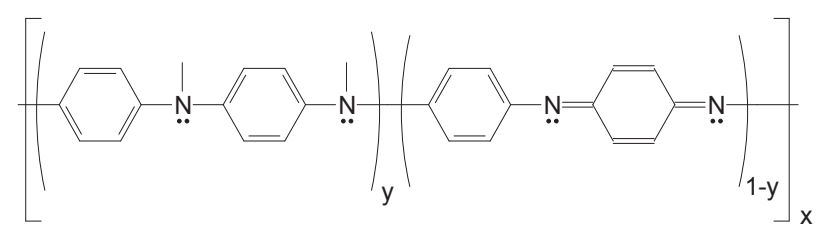

$y$ corresponde à fração de unidades reduzidas e (1-y) a fração de unidades repetitivas oxidadas. $\mathrm{O}$ valor de $y$ pode variar continuamente entre 0 e 1 . Quando $y$ assume o valor de 1 , obtém-se o polímero completamente reduzido e apresentando apenas nitrogênio amina. Tal forma isolante é conhecida como leucoesmeraldina. (FAEZ et al., 2000).

A PAni é um polímero cujas características físicas e químicas dependem fortemente do método de preparação e dos parâmetros experimentais de síntese. Sabe-se que a morfologia, a condutividade e outras propriedades dependem do contra-íon incorporado, bem como do grau de cristalinidade, do procedimento de preparação (MATTOSO, 1996).

A PAni sintetizada eletroquimicamente consiste em dois tipos de camadas. A primeira é formada nos estágios iniciais de crescimento e apresenta uma estrutura mais compacta, enquanto que a segunda apresenta uma estrutura menos densa. (BAZITO, 2002, CÓRDOVA et al., 1994). As investigações das eletropolimerizações dos filmes de PAni nos estágios iniciais têm sido reportadas por vários pesquisadores (BARBERO, 1994, MOTHEO et al., 1998, KANATZIDIS, 1990, SAMUI et al., 2003).

A eletrodeposição da PAni inclui um processo de nucleação similar ao processo de nucleação da deposição de um metal. Acontece nos estágios iniciais de crescimento do filme e obedecendo a uma nucleação progressiva $2 \mathrm{D}$ a um mecanismo 3D em ácido sulfúrico e/ou 2D em ácido perclórico como eletrólito suporte (KANG et al., 1998). A maioria dos modelos desenvolvidos para descrever os processos de nucleação e crescimento de filmes eletrodepositados visa à obtenção de uma expressão analítica para a descrição dos transientes de corrente (curvas de densidade de corrente vs. tempo) (SCHARIFKER et al., 1998). Um modelo de NCS (Nucleação-Crescimento-Superposição) foi desenvolvido por (Scharifker., 1998), que obteve expressões analíticas para reações de eletrodeposição de metais, polímeros condutores, em soluções aquosas, limitadas por difusão. As suposições desse modelo impõem algumas restrições para uma descrição geral dos mecanismos de nucleação e crescimento em células eletroquímicas.

O modelo de Scharifker assume a existência de sítios ativos (locais onde pode ocorrer a formação de núcleos), cuja densidade de núcleos formados (N) pode ser descrita por,

$$
N(t)=N_{\infty}[1-\exp (-A t)]
$$

onde $N_{\infty}$ é a densidade de sítios, $t$ é o tempo de eletrodeposição e $A$ é a taxa de nucleação.

$\mathrm{Na}$ Figura 2 encontram-se zonas de difusão geradas em torno de núcleos formados na superfície do eletrodo e que estão em fase de crescimento.

Figura 2 - (a) Representação esquemática do crescimento das zonas de difusão e suas eventuais sobreposições; (b) Vista plana de núcleos hemisféricos, aleatoriamente distribuídos na superfície do eletrodo, os círculos representam as zonas de difusão (SCHARIFKER et al., 1998).

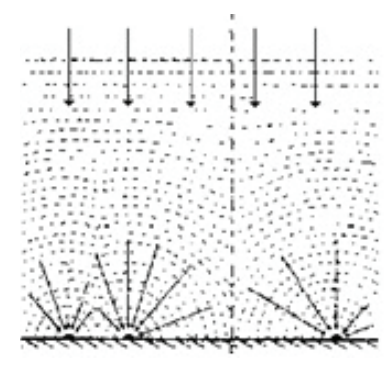

A

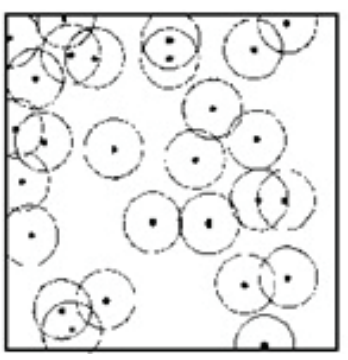

B

Publ. UEPG Exact Earth Sci., Agr. Sci. Eng., Ponta Grossa, 18 (1): 15-21, jan/jul. 2012

Disponivel em <http://www.revistas2.uepg.br/index.php/exatas> 
Para valores finitos de $t$ e $A$ o limite de $N$ será o da sobreposição das zonas de difusão, que são provenientes do gradiente de concentração que se forma em torno de cada núcleo. A taxa de transferência de carga para a reação que acontece nas eletrodeposições metálicas é suficientemente alta para favorecer o crescimento continuado de núcleos estabelecidos. Após a formação inicial destes núcleos, admitindo-se que os mesmos cresçam sem superposição, e sobre condições de controle de transferência de carga, a densidade de corrente para um número fixo de núcleos $N$, formada instantaneamente na superfície do eletrodo em $t=0$ pela aplicação de um potencial externo é dada por:

$$
J_{i}(t)=z F \pi(2 D c)^{\frac{3}{2}}\left(\frac{M}{\rho}\right)^{\frac{1}{2}} N t^{\frac{1}{2}}
$$

onde $z F$ é a carga molar da espécie depositada, $D$ é o coeficiente de difusão em $\mathrm{cm}^{2} \mathrm{~s}^{-1}, c$ é a concentração em $\mathrm{mol} \mathrm{cm}^{-3}, M$ é a massa molar e $\rho$ é a densidade de material depositado. A dependência linear de $J$ com $t^{1 / 2}$ é frequentemente observada não só para grãos de forma hemisférica, mas também para outras morfologias devido ao fato de que as zonas de difusão em torno dos núcleos avançam radialmente de maneira muito mais rápida que o perímetro dos mesmos. Isto faz com que o fluxo de massa tenha simetria hemisférica (GUNAWARDENA et al., 1982).

Se a taxa com que os núcleos são formados na superfície não for instantânea, ou seja, no caso de nucleação progressiva, novos núcleos são formados continuamente, e a densidade de corrente será dada por,

$$
J_{p}(t)=\frac{2 z F \pi A N_{\infty}(2 D c)^{\frac{3}{2}} M^{\frac{1}{2}} t^{\frac{3}{2}}}{3 \rho^{\frac{1}{2}}}
$$

As expressões para densidade de corrente para os dois tipos de nucleação (2 e 3) são restritas para casos em que os núcleos crescem independentemente, o que não ocorre na realidade devido à sobreposição das zonas de difusão de vários núcleos próximos (SCHARIFKER et al., 1998).
Sharifker considerou um conjunto de núcleos hemisféricos distribuídos aleatoriamente na superfície do eletrodo, bem como o crescimento controlado por difusão. Cada núcleo desenvolve uma zona de difusão hemisférica que cresce com uma velocidade radial de forma que as zonas de difusão comecem a se sobrepor.

Devido ao fato de os núcleos estarem distribuídos aleatoriamente, existe a possibilidade de haver superposição. A fração de área coberta por zonas de difusão, $\theta$, pode ser obtida pelo teorema de Avrami (1939), que considera que os núcleos crescem em uma determinada direção à velocidade constante.

$$
\theta_{\mathrm{i}}=1-\exp (-\mathrm{N} \pi \mathrm{KDt})
$$

A conservação de massa requer que a quantidade de material introduzido nas zonas de difusão seja igual à quantidade incorporada no núcleo crescido. Desse modo, a densidade de corrente para a condição de difusão planar em direção à superfície do eletrodo é dada por:

$$
J=z F C\left(\frac{D}{\pi t}\right)^{\frac{1}{2}} \theta
$$

Das equações (4) e (5) obtêm-se para a nucleação instantânea.

$J=z F c\left(\frac{D}{\pi t}\right)^{\frac{1}{2}}[1-\exp (-\mathrm{N} \pi \mathrm{KDt})]$ onde $\mathrm{k}=\left(\frac{8 \pi \mathrm{Mc}}{\rho}\right)^{\frac{1}{2}}$

No entanto, se a nucleação for progressiva, $\mathrm{N}(t)=$ $A N_{\infty} t$ e, a área coberta pelas zonas de difusão será:

$$
\theta_{\pi}=1-\exp \left(\frac{-A N_{\infty} \pi K^{\prime} D t^{2}}{2}\right)
$$

Substituindo a equação 7 em 5, obtém-se:

$$
\begin{aligned}
& J_{p}=z F C\left(\frac{D}{\pi t}\right)^{\frac{1}{2}}\left[1-\exp \left(\frac{-A N_{\infty} \pi k t^{2}}{2}\right)\right] \\
& \text { onde } \quad k^{\prime}=\frac{4}{3}\left(\frac{8 \pi M c}{\rho}\right)^{\frac{1}{2}}
\end{aligned}
$$


A equação 8 refere-se à densidade de corrente para a nucleação progressiva. Para comparar os resultados teóricos com os dados experimentais, determina-se a densidade de corrente máxima, $\mathrm{J}_{\max }$ e o tempo máximo $t_{\max }$ por meio da primeira derivada de $J$, em relação a $t$, igualando a zero. Assim, obtém-se:

Figura 3 - Gráfico adimensional das variáveis normalizadas para nucleação a) instantânea e b) progressiva (SCHARIFKER et al., 1998).

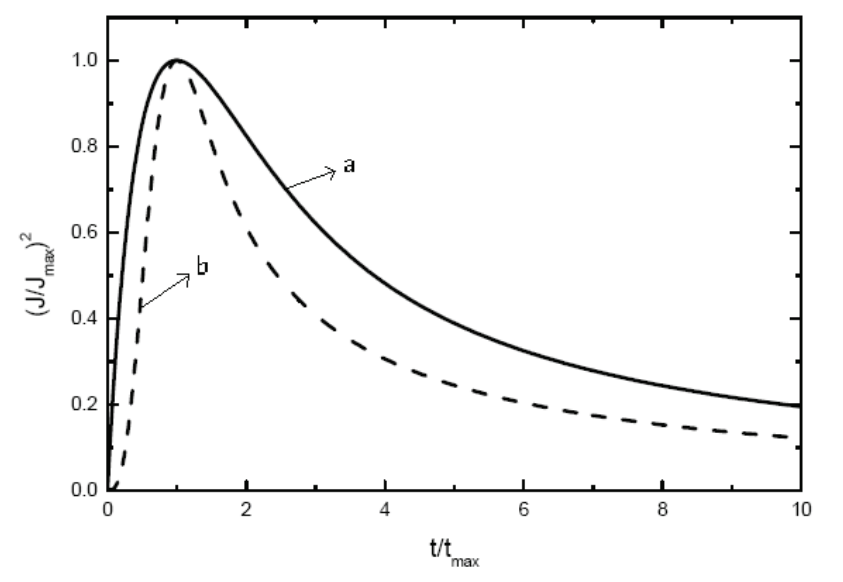

$$
t_{\max }=\left(\frac{4,6733}{A N_{\infty} \pi K^{\prime} D}\right)^{\frac{1}{2}}
$$$$
J_{\max }=0,4615 z F c D^{\frac{3}{2}}\left(K^{\prime} A N_{\infty}\right)^{\frac{1}{4}}
$$

$$
\begin{gathered}
\mathrm{e} \\
t_{\max }=\frac{1,564}{N \pi K D} \\
J_{\max }=0,6382 z F c D(K N)^{\frac{1}{2}}
\end{gathered}
$$

para a nucleação progressiva e instantânea, respectivamente. Dessa forma, chega-se à expressão duplamente normalizada para a nucleação instantânea:

$$
J^{\prime 2}=1,9542\left(t^{\prime}\right)^{-1}\left[1-\exp \left(-1,2564 t^{\prime}\right)\right]^{2}
$$

Para a nucleação progressiva:

$$
J^{\prime 2}=1,2254\left(t^{\prime}\right)^{-1}\left[1-\exp \left(-2,3367 t^{\prime 2}\right)\right]^{2}
$$

E ambas as expressões

$$
J^{\prime}=\frac{J}{J_{\max }} \quad \text { e } \quad t^{\prime}=\frac{t}{t_{\max }}
$$

É importante notar que essas expressões obtidas por Scharifker (1998) não dependem dos parâmetros de deposição ou do sistema com taxas de nucleação. Na Figura 3, são representadas as curvas referentes às equações 11 e 12, as quais podem ser comparadas diretamente com os resultados experimentais.

\section{Materiais e Métodos.}

A liga de aço inox 304 com composição de $20 \%$ de cromo e $15 \%$ de níquel foi cortada nas dimensões de $0,5 \mathrm{~cm}$ de diâmetro por $0,3 \mathrm{~cm}$ de espessura. Antes de todos os ensaios a amostra foi lixada mecanicamente nas granulometrias 120, 220, 320, 400, 600 e 1200. Após o lixamento feito com uma lixa de granulometria 1200, a amostra foi desengraxada em solução de álcool etílico em banho de ultrassom por 15 minutos.

Os filmes de PAni foram sintetizados sobre a liga de aço inox 304 por meio de de deposições potenciostáticas, as quais foram realizadas a 0,40 ; 0,$60 ; 0,70 ; 0,75$ e $0,80 \mathrm{~V}$ versus eletrodo de $\mathrm{Ag} / \mathrm{AgCl}$ durante 25 minutos, a partir do meio:

- ácido sulfúrico $0,5 \mathrm{~mol} \mathrm{~L}^{-1}$ e anilina $0,20 \mathrm{~mol} \mathrm{~L}^{-1}$

As curvas obtidas foram registradas com o auxílio de um potenciostato/autolab PGSTAT302N 30 $\mathrm{V} / 2000 \mathrm{~mA}$. Estas deposições seguem um mesmo padrão: inicialmente há o carregamento da dupla camada, com um aumento brusco de corrente seguido de um decréscimo de corrente relativo ao período de indução, período no qual não há a formação de PAni. Após essas etapas iniciais, ocorre o início da eletropolimerização da anilina, formando o filme de PAni sobre o eletrodo.

Para o tratamento matemático de nucleação do filme depositado utilizou-se a técnica de deposição potenciostática com a aplicação dos potenciais de $0,80,0,75$ e $0,70 \mathrm{~V}$ versus eletrodo de $\mathrm{Ag} / \mathrm{AgCl}$.

\section{Célula eletroquímica.}

Os eletrodos de trabalho consistem nas respectivas ligas de aço inox 304 . O contra-eletrodo utilizado foi uma chapa de platina de dimensões de $2,0 \mathrm{~cm} \times 2,0 \mathrm{~cm}$. O eletrodo de referência utilizado em todos os experimentos foi de $\mathrm{Ag} / \mathrm{AgCl}$. Os eletrodos de trabalho utilizados foram embutidos em resina cristal e tinham área exposta de $0,39 \mathrm{~cm}^{2}$. 
O copo dessa célula é constituído de vidro e sua tampa é confeccionada em PVC.

\section{Resultados e Discussão.}

O estágio de crescimento do filme polimérico é evidenciado pelo aumento de corrente, gerado pela oxidação das moléculas de anilina. Quando a corrente atinge um patamar no qual se mantém estável, a taxa de eletropolimerização é constante. Nesse tipo de deposição, o período de indução é dependente da concentração do monômero e do potencial aplicado, sendo maior para baixas concentrações de monômeros e baixos potenciais. Nessas condições há pouca geração de cátions fazendo com que a taxa de deposição do filme polimérico seja pequena (BARBERO et al., 1994; MOTHEO et al., 1998).

A curva da corrente em função do tempo apresentada na Figura 4 mostra o comportamento característico para a deposição de polianilina sobre aço inox 304. No início da deposição há um aumento de corrente devido ao carregamento da dupla camada, seguido por uma queda de corrente referente ao tempo de indução. Após a queda, a corrente atinge um patamar, no qual ocorre a oxidação das moléculas de anilina e chega-se a uma taxa constante de deposição de PAni. O meio eletrolítico e a concentração do monômero, no qual ocorre a polimerização, influenciam no tempo de indução e no patamar de corrente obtido para a deposição do filme polimérico (CORDOVA et al., 1994).

Figura 4 - Curva de corrente em função do tempo obtida durante a deposição do filme polimérico sobre a liga de aço inox 304, a partir de solução de $\mathrm{H}_{2} \mathrm{SO}_{4} 0,5 \mathrm{Mol} \mathrm{L}^{-1}$ e anilina a $0,2 \mathrm{Mol} \mathrm{L}^{-1}$ a vários potenciais.

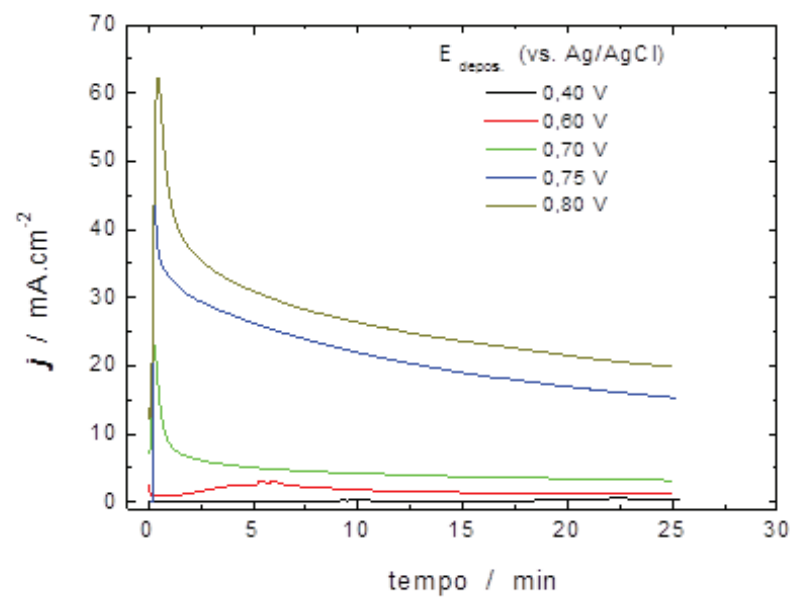

\section{Nucleação do crescimento do filme de Polianilina}

O meio eletrolítico e o potencial de crescimento do filme, no qual ocorre a polimerização, influenciam no tempo de indução, bem como no patamar de corrente obtido para a deposição do filme polimérico. Pela análise das curvas da Figura 4 é possível observar que houve a formação do filme por meio do potencial de $0,70 \mathrm{~V}$. Em outros potenciais mais altos é possível observar um aumento significativo da corrente, oque indica um filme mais espesso, em relação ao filme obtido sobre potencial de $0,70 \mathrm{~V}$ versus $\mathrm{Ag} / \mathrm{AgCl}$. A Figura 5 mostra as curvas obtidas para o tratamento matemático com base nas equações 11 e 12 para a deposição do filme de PAni realizada em potencial de $0,80 \mathrm{~V}$ versus $\mathrm{Ag} / \mathrm{AgCl}$.

A curva (a) da Figura 5 apresenta um formato muito parecido com a curva teórica obtida para um processo instantâneo, o que leva a crer que, o comportamento de nucleação para o filme de PAni apresenta-se também como um processo instantâneo, ou seja, o filme começa a se formar na superfície da liga logo nos primeiros segundos de aplicação do potencial. Posteriormente, percebe-se uma taxa de deposição constante, o que indica um processo de crescimento rápido do filme polimérico em que cada núcleo tende a ser controlado por difusão. (CORDOVA et al., 1994; SCHARIFKER et al., 1998; HILLS et al., 1974; GUNAWARDENA et al., 1982).

Figura 5 - Curvas para tratamento de NCS referente à liga de aço inox 304 utilizando as equações (11) e (12) de Scharifker e Hills (a) Curva experimental sem tratamento matemático; (b) Nucleação Instantânea; (c) Nucleação Progressiva.

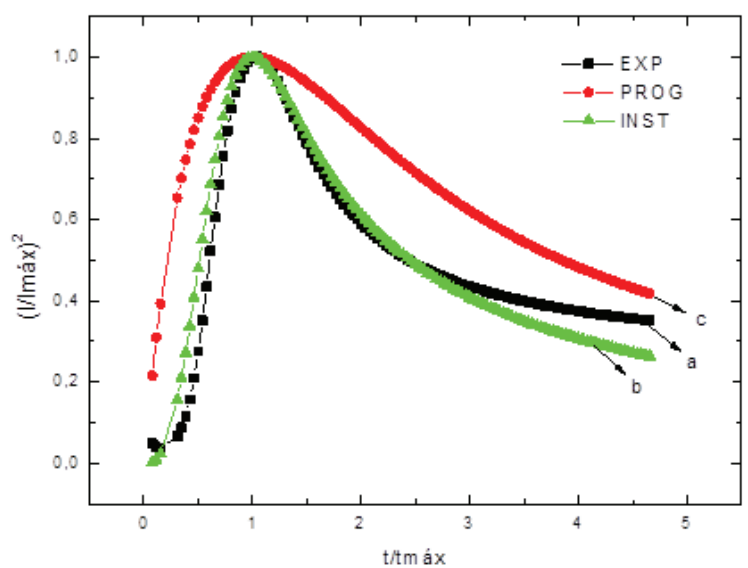


A Figura 6 mostra as curvas obtidas para o tratamento matemático utilizando as equações $11 \mathrm{e}$ 12 para a deposição do filme de PAni realizada em potencial de $0,75 \mathrm{~V}$ versus $\mathrm{Ag} / \mathrm{AgCl}$. Nota-se que a curva experimental, $(a)$, não é parecida com a curva (a) da Figura 5. No entanto no tempo inicial de sua formação até o tempo 1,1 no eixo das abscissas, a curva (a) da Figura 6 se semelhança à curva (b) da mesma que apresenta um processo de nucleação instantânea. Isso leva a crer que o filme de PAni se deposita logo nos primeiros instantes de aplicação do potencial de $0,75 \mathrm{~V}$ versus $\mathrm{Ag} / \mathrm{AgCl}$.

Figura 6 - Curva para tratamento de NCS referente à liga de aço inox 304 utilizando as equações (11) e (12) de Scharifker e Hills (a) Curva experimental sem tratamento matemático; (b) Nucleação Instantânea; (c) Nucleação Progressiva.

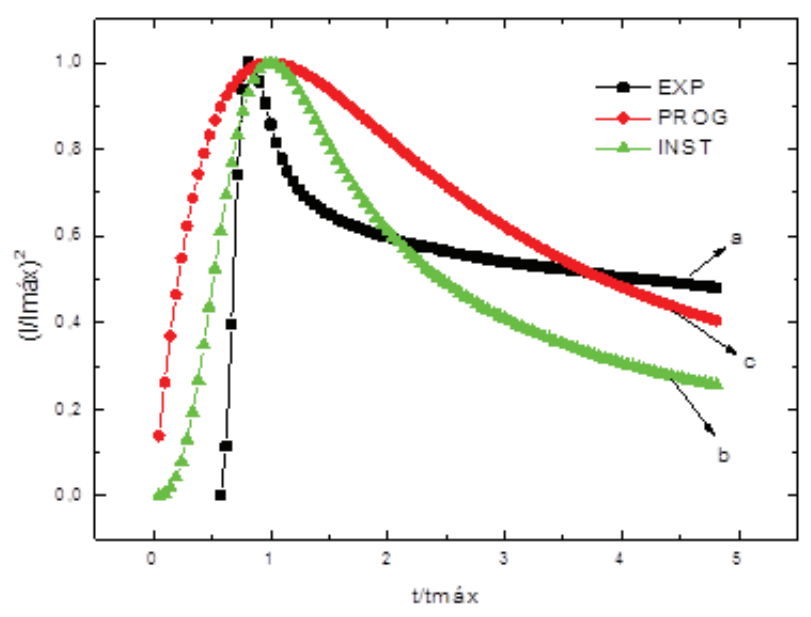

Na Figura 7, é observado que a curva experimental (a) se assemelha à curva (b), que apresenta um tratamento matemático de nucleação para um processo instantâneo, ou seja, o crescimento do filme também acontece logo nos primeiros instantes de aplicação do potencial de $0,70 \mathrm{~V}$ versus eletrodo de $\mathrm{Ag} / \mathrm{AgCl}$.
Figura 7 - Curva para tratamento de NCS referente à liga de aço inox 304 utilizando as equações (11) e (12) de Scharifker e Hills (a) Curva experimental sem tratamento matemático; (b) Nucleação Instantânea; (c) Nucleação Progressiva.

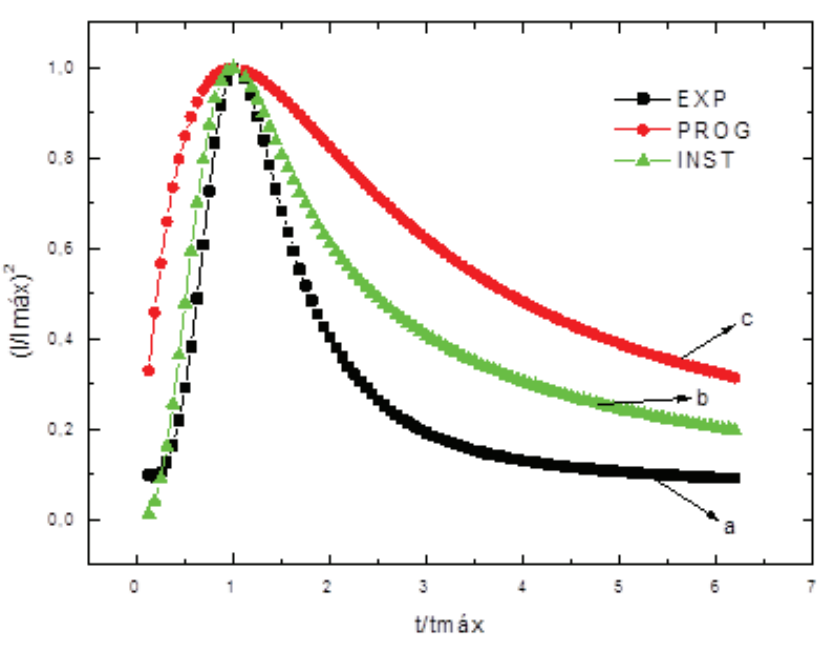

\section{Conclusões.}

O estudo potenciostático mostra que no caso dessa pesquisa, há apenas um estágio de crescimento para a PAni. A possibilidade de se observar um estágio em particular depende do contra-íon incorporado, da concentração do monômero anilina e do potencial de síntese utilizado. Todos esses fatores são responsáveis pela natureza do filme do polímero formado sobre o substrato. Com relação à liga de aço inox 304 as curvas obtidas para os tratamentos de NCS, Nucleação-Crescimento-Superposição, apresentaram um formato muito semelhante às curvas teóricas do processo denominado instantâneo. Nos primeiros instantes de aplicação do potencial, o filme começa a formar núcleos de deposição e, logo em seguida, é possível observar o crescimento do filme sobre a liga estudada.

\section{Referências}

AVRAMI, M., Kinetics of phase change. I, Journal of Chemical Physics, v.7, p. 1103-1112, 1939.

BARBERO, C., Sulfonated polyaniline films as cation insertion electrodes for battery applications, J. Mater. Chem., n. 4, p. 1775, 1994.

BAZITO, F.C., Estudo espectroeletroquímico de um copolímero alternado de PAni e PPS, Tese de Doutorado - Instituto de Química - Universidade de São Paulo, 2002. 
CORDOVA, R.; del VALLE, M.A.; ARRATIA, A.; GÓMEZ, H.; SCHREBLER, R. Effect of anions on the nucleation and growth mechanism of polyaniline, Journal of Electroanalytical Chemisty, n. 377 p. 75-83 1994.

FAEZ, R.; REIS C.; SCANDIUCCI, P.; KOSIMA, O.K.; RUGGERI, G.; De PAOLI, M.A. Polímeros condutores, Química Nova na Escola., n. 11, 2000.

GUNAWARDENA, G.; HILLS, G.; MONTENEGRO, I.; SCHARIFKER, B. Electrochemical nucleation: Part I. General considerations. Journal of Electroanalytical Chemistry, n. 138, p. 225, 1982.

HILLS, G. J.; SCHIFFRIM, D. J.; THOMPSON, J. Electrochemical nucleation from molten salts - I. Diffusion controlled electrodeposition of silver from alkali molten nitrates. Electrochimica Acta, n. 19, p. 657, 1974.

KANATZIDIS, M.G. Conductive polymers. Americal Chemical Society, n.68, p.36, 1990.

KANG, E. T.; NEOH, K. G.; TAN, K. L. Polyaniline: a polymer with many interesting intrinsic redox states. Progress in Polymer Science, n. 23, p. 277, 1998.

MATTOSO, C.L.H. Polianilinas, síntese estrutura e propriedades, Química Nova, n. 19, p. 4, 1996.

MOTHEO, A.J.; SANTOS JUNIOR, J.R.; VENANCIO, E.C.; MATTOSO, L.H.C.; Influence of different types of acidic dopant on the electrodeposition and properties of polyaniline films. Polymer, n. 39, p. 6977, 1998.

SAMUI, A. B.; PATANKAR, A. S.; RANGARAJAN, J.; DEB, P.C. Study of polyaniline containing paint for corrosion prevention. Progress in Organic Coatings, $\mathrm{n}$. 47, p. 1, 2003.

SCHARIFKER, B.; HILLS, G. Theoretical and experimental studies of multiple nucleation. Electrochimica Acta, n. 28, p. $879,1983$.

Recebido em 25/02/2013

Aceito em 17/04/2013 